\title{
Note sur le comportement sexuel des juments en milieu tropical
}

\author{
par P. REDON et L, FAYOLLE
}

La conduite d'un Centre d'Elevage suppose, pour être efficace, une connaissance approfondie du cycle sexuel de ses pensionnaires. Cette activité n'admet, en particulier, aucune ignorance lorsqu'elle s'adresse aux juments, femelles dont la fécondation est facilement compromise par une monte en main mal conduite. En effet, le moment optimum de l'acte ne peut être rationnellement déterminé que si des chiffres sans équivoque concrétisent, dans l'esprit du responsable, durée de gestation, cestrus, interœstrus, délai de rut après mise-bas, etc...

Le relevé et l'étude des observations enregistrées - au Centre d'Elevage de Dahra (zone sahélienne du Sénégal) de 1952 à 1955 a eu justement pour but cette definition précise du comportement sexuel - des juments en milieu tropical.

\section{I. - MATÉRIEL D'EXPÉRIENCE}

Les observations ont porté sur une population de 24 juments se répartissant en 18 juments locales et 6 juments Arabe-Barbe.

Juments locales

Ces juments appartiennent à la race dite " du fleuve " et se caractérisent essentiellement par une taille élevée $(1,40 \mathrm{~m}$ à $1,45 \mathrm{~m})$, alors que les autres races sénégalaises plafonnent à $1,38 \mathrm{~m}$ (M'Bayars) et 1,33 $\mathrm{m}$ (M'Pars).

Comme la plupart des races aoéfiennes, cette population trouve son origine dans des croisements entre le cheval africain initial et les coursiers des conquérants berbères et arabes, qui, à diverses époques, traversèrent un Sahara moins aride qu'aujourd'hui.

Le cheval initial était un poney peu différent au Sénégal du « M'par » actuel.

- L'influence arabe et barbe sur la population équine du Territoire est conditionnée : lo Par le passé historique : très nette au bord du Sénégal qui vit passer au $\mathrm{IX}^{\mathrm{c}}$ siècle les derniers Zenega de l'Empire de Ghana, peut-être au $\mathrm{X}^{\mathrm{e}}$ les Almoravides, au XVIo les Dawi Hassan, elle s'estompe vers le Sud où la tsé-tsé démontait les cavaliers.

$2^{n}$ Par l'épidémiologie qui impose un fort pourcentage de sang poney, seul garant de survie en pays à trypanosomiases et peste équine enzootiques (Sine-Saloum - Côte Atlantique).

$3^{\circ}$ Par des conditions alimentaires, limitant aux seules régions productrices de mil, l'élevage du cheval de grande taille (région du fleuve).

$4^{\circ}$ Par les courants commerciaux actuels :

Le cheptel équin de Bakel (Haut-Sénégal) subit l'influence des chevaux soudanais fortement berbérisés, qui traversent ses gués pour être vendus au Sénégal.

Le moyen fleuve (de Podor à Matam) reste en relations plus ou moins lointaines avec l'Afrique du Nord, par l'intermédiaire des Maures du Hodh et du Tagant.

Toutes ces considérations permettent de garantir chez nos juments achetées sur le fleuve (régions de Matam et de Bakel), la présence d'un fort pourcentage de sang arabe et barbe.

Leurs éléments métriques moyens sont les suivants :

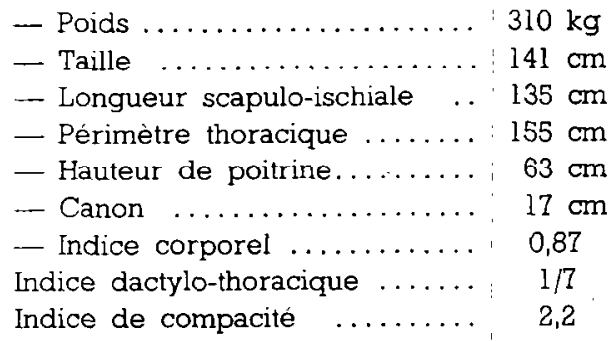

Juments Arabe-Barbe

Importées d'Afrique du Nord (Maroc) en 1952. 
Toutes ces juments, entretenues d'une manière relativement extensive (pacage la nuit en parc clos - complément alimentaire concentré le jour en paddok), sont en bon état d'entretien.

\section{II. - PROTOCOLE DES OBSERVATIONS}

Le dépistage des juments en chaleur se fait par la méthode classique du « souffleur».

Un étalon est passé chaque matin dans un colloir d'accès au box des juments. Le procédé est pratique et suffit, dans la plupart des cas, à détecter l'état d'œstrus.

La jument en chaleur vient généralement audevant de l'étalon à travers les, barreaux du box et se laisse facilement flairer par: lui, tout en présentant les signes classiques de l'cestrus (position « campée », manifestations vulvo-vésicales, etc...). En cas de doute, et, notamment chez les sujets dont les manifestations demeurent plus ou moins confuses, la jument est alors conduite à la barre et quelquefois même soumise aux entraves en vue d'un examen plus complet.

Sur les juments vides, les investigations commencent trois mois avant l'ouverture de la saison de monte fixée, à Dahra, pour des raisons alimentaires, au ler juillet. Une telle politique permet de bien connaître le cycle de ces juments et, l'hivernage venu, de les livrer à la saillie avec un maximum de chances.

Les juments suitées sont présentées au « souffleur » le $3^{\mathrm{e}}$ jour suivant la mise-bas.

Chaque jument possède une fiche individuelle où sont notées les cycles successifs et les réactions observées.

\section{III. - RÉSULTATS}

\section{Réaction des juments en présence de l'étalon.}

Le comportement est, dans l'ensemble, normal (acceptation en période d'œstrus avec manifestations vulvo-vésicales; réactions de défense en période d'ancestrus).

Certains sujets (le fait a été observé sur 3 d'entre eux) sont du type émotif (réaction de défense et manifestations vulvo-vésicales à l'œstrus comme à l'ancestrus).

Le type passif n'a pas été observé $\left({ }^{*}\right)$.

(*) Bertelon, - Notes pratiques de gynécologie équine. Rev. Méd. Vét. (1951), 14, février.

\section{Apparition de la puberté.}

Les chaleurs apparaissent vers l'âge de 7 à 8 mois, tant sur les pouliches du pays que sur les pouliches de races améliorées (demi-sang et arabe-barbe). C'est en effet vers cette période, alors que mâles et femelles vivent encore en communauté, qu'il est possible de remarquer les premières manifestations. sexuelles.

Ces observations très fragmentaires n'ont pas permis l'étude statistique du phénomène:

\section{Influence de la saison sur l’activité générale.}

De même que dans l'étude précédente, les renseignements recueillis à ce sujet sont encore insuffisants pour justifier une interprétation statistique. Une première série d'observations (5 sujets examinés sur un cycle de onze mois consécutifs : janvier à novembre) permet toutefois de supposer que les juments élevées en milieu tropical ont un type sexuel à caractère continu. Tous les individus soumis à l'expérience présentèrent en effet, une activité génitale constante et parfaitement régulière tout au long de la période d'observations.

\section{Durée de l'cestrus et influence raciale.}

TABLEAU 1

\begin{tabular}{|c|c|c|}
\hline $\begin{array}{c}\text { Durée des } \\
\text { chaleurs } \\
\text { (en jours) }\end{array}$ & \multicolumn{2}{|c|}{ Nombre d'observations } \\
\cline { 2 - 3 } 12 & 1 & Arabe-Barbe \\
\hline 11 & 1 & \\
10 & 2 & 1 \\
9 & 3 & 6 \\
8 & 26 & 12 \\
7 & 35 & 16 \\
6 & 27 & 6 \\
5 & 21 & 51 \\
4 & 13 & 10 \\
\hline 3 & 6 & \\
\hline Total & 135 & \\
\hline
\end{tabular}

Ont été exclus des observations trois cas d'hyperœstrus (deux sur des juments locales de 21 et 54 jours, un sur une jument arabe-barbe de 24 jours) consi dérés comme pathologiques.

La durée moyenne de l'œstrus est chez les juments indigènes de $6,3 \pm 0,3$ jours et chez les arabe-barbe de 6,1 $\pm 0,3$ jours. Les différences enregistrées ne sont pas significatives. 


\section{Influence de l'âge sur la duxée des chaleurs.}

TABLEAU II

\begin{tabular}{|c|c|c|c|c|c|c|}
\hline \multirow{3}{*}{$\begin{array}{l}\text { Durée } \\
\text { des } \\
\text { chaleurs }\end{array}$} & \multicolumn{6}{|c|}{ NOMBRE D'OBSERVATIONS } \\
\hline & \multicolumn{2}{|c|}{ Juments de 3 à 4 ans } & \multicolumn{2}{|c|}{ Juments de 5 à 7 ans } & \multicolumn{2}{|c|}{$\begin{array}{l}\text { Juments de } 8 \text { ans } \\
\text { et au-dessus }\end{array}$} \\
\hline & Locales & Arabe-Barbe & Locales & Arabe-Barbe & Locales & Arabe-Barbe \\
\hline 12 & & & & & 1 & Aucune ob- \\
\hline 11 & & & 1 & & & servation, \\
\hline 10 & & & 2 & & & trop jeu- \\
\hline 9 & 1 & & $!$ & 1 & 1 & nes. \\
\hline 8 & 5 & 5 & 10 & 1 & 11 & \\
\hline 7 & 8 & 8 & 14 & 4 & 11 & \\
\hline 6 & 7 & 9 & 14 & 7 & 6 & \\
\hline 5 & 6 & 2 & 10 & 8 & 5 & \\
\hline 4 & 1 & & 9 & 6 & 3 & \\
\hline 3 & & & 5 & & $i$ & \\
\hline Total & 28 & 24 & 66 & 27 & 39 & \\
\hline
\end{tabular}

L'étude de ce tableau montre que l'âge n'a pas d'action déterminante sur la durée des chaleurs.
L'interprétation statistique donne, en effet, les résultats suivants :

TABLEAU III

- INTERPRETATION STATISTIQUE DE LA DUREE DES CHALEURS -

Moyenne en jourg

Erreur de la moyeme

\begin{tabular}{|c|c|c|c|c|c|}
\hline \multicolumn{3}{|c|}{ Juments locales } & \multicolumn{3}{c|}{ Juments Arabe-Barbe } \\
\hline 3 à 4 ans & 5 à 7 ans & 8 ans et + & 3 à 4 ans & 5 à 7 ans & 8 ans et + \\
\hline & 6,0 & 6,7 & 6,6 & 5,6 & - \\
0,4 & 6,0 & 0,5 & 0,4 & 0,8 & - \\
0,5 & 0,4 & & \multicolumn{3}{|l}{} \\
\hline
\end{tabular}


TABLEAU IV

\begin{tabular}{|c|c|c|}
\hline \multirow{2}{*}{$\begin{array}{c}\text { Irurée } \\
\text { interoestrale } \\
\text { (en jours) }\end{array}$} & \multicolumn{2}{|c|}{ Nombre d'observations } \\
\hline & Juments Iocales & Arabe-Barbe \\
\hline 10 & 4 & \\
\hline 11 & 3 & \\
\hline 12 & & 2 \\
\hline $13^{\prime}$ & 5 & \\
\hline 14 & 5 & 6 \\
\hline 15 & 13 & 5 \\
\hline 16 & 5 & 3 \\
\hline 17 & 5 & 2 \\
\hline 18 & 10 & 1 \\
\hline 19 & 4 & 1 \\
\hline 20 & 5 & \\
\hline 21 & 4 & \\
\hline 22 & 1 & 1 \\
\hline 23 & 1 & .1 \\
\hline 24 & & 1 \\
\hline Total & 65 & 23 \\
\hline
\end{tabular}

6. Durée de l'intercestrus et influence raciale.

De même que pour la durée dess chaleurs, le comportement des deux races est sensiblement la même. La durée moyenne intercestrale enregistrée est de $16,3 \pm 0,8$ jours pour les juments du pays et $15,8 \pm 1,3$ pour les Arabe-Barbe.

7. Influence de l'âge sur la durée de l'intercestrus.

TABLEAU

\begin{tabular}{|c|c|c|c|c|c|c|}
\hline \multirow{3}{*}{$\begin{array}{c}\text { Durée } \\
\text { interoestrale } \\
\text { (jours) }\end{array}$} & \multicolumn{6}{|c|}{ MOMBRE D'OBSERVATIONS } \\
\hline & \multicolumn{2}{|c|}{ Juments de 3 à 4 ans } & \multicolumn{2}{|c|}{ Juments de 5 à 7 ans } & \multicolumn{2}{|c|}{$\begin{array}{l}\text { Juments de } 8 \text { ans } \\
\text { et au-dessus }\end{array}$} \\
\hline & Loceles & Arabe-Barbe & Locales & Arabe-Barbe & Locales & Arabe-Barbe \\
\hline 24 & 1 & & 1 & . & & \\
\hline 23 & & & & 1 & & \\
\hline 22 & & & 1 & & & \\
\hline 21 & 1 & & & $\mathfrak{i}$ & & \\
\hline 20 & 1 & & 4 & 1 & 1 & \\
\hline 19 & & 1 & 2 & & 1 & : \\
\hline 18 & 4 & 1 & 5 & & 2 & \\
\hline 17 & $\mathbf{1}$ & 1 & 3 & & & \\
\hline 16 & 1 & 1 & 3 & 1 & 1 & \\
\hline 15 & 2 & 3 & 6 & 2 & 4 & \\
\hline 14 & $!$ & 4 & 4 & 2 & $i$ & \\
\hline 13 & 2 & & 2 & 2 & 1 & \\
\hline 12 & & 2 & & & & \\
\hline 11 & & & 1 & & 2 & \\
\hline 10 & 1 & & 1 & 2 & 2 & \\
\hline Total & 15 & 13 & 33 & 12 & 15 & \\
\hline
\end{tabular}


Résultats

TABLEAU VI

\begin{tabular}{|l|c|c|c|c|c|c|}
\hline \multirow{2}{*}{} & \multicolumn{4}{|c|}{ Juments locales } & \multicolumn{3}{|c|}{ Juments Arabe-Barbe } \\
\cline { 2 - 7 } & 3 a 4 ans & 5 a 7 ans & 8 ans et + & 3 à 4 ans & 5 à 7 ans & 8 ans et + \\
\hline Moyenne en jours & 16,6 & 16,5 & 14,7 & 15,0 & 15,3 & 14,7 \\
Erreur de la moyenne & 0,8 & 1,1 & 1,7 & 1,3 & 2,3 & 1,7 \\
\hline
\end{tabular}

Une fois de plus les différences observées ne sont pas significatives et ne permettent pas de considérer l'âge comme un facteur susceptible d'influencer la durée intercestrale.

8. Duree de la gestation et influence raciale.

TABLEAU VII

\begin{tabular}{|c|c|c|}
\hline \multirow{2}{*}{$\begin{array}{l}\text { Durée de la } \\
\text { gestation }\end{array}$} & \multicolumn{2}{|c|}{ Nombre d'observations } \\
\hline & Junents locales & Arabe-Barbe \\
\hline 314 & 2 & \\
\hline 316 & 1 & \\
\hline 319 & 2 & \\
\hline 320 & 2 & \\
\hline 321 & 1 & \\
\hline 323 & 1 & \\
\hline 324 & 1 & \\
\hline 325 & 1 & 1 \\
\hline 326 & 1 & \\
\hline 327 & 1 & \\
\hline 328 & 3 & 1 \\
\hline 329 & 2 & 2 \\
\hline 330 & 2 & 1 \\
\hline 331 & 2 & 1 \\
\hline 332 & 3 & 2 \\
\hline 333 & 1 & 1 \\
\hline 334 & 1 & 1 \\
\hline 335 & 2 & \\
\hline 336 & 2 & 1 \\
\hline 337 & 2 & \\
\hline 338 & 3 & \\
\hline 340 & 1 & \\
\hline 341 & 1 & \\
\hline 343 & 1 & 2 \\
\hline 344 & 2 & \\
\hline 346 & 1 & \\
\hline 348 & 1 & \\
\hline Total & 43 & 13 \\
\hline
\end{tabular}

Le facteur racial n'intervient pas dans la durée de la gestation, les différences enregistrées n'étant pas significatives. La durée moyenne est. chez les juments locales, de $331,2 \pm 2,5$ jours et, chez les juments Arabe-Barbe, de 333,7 $\pm 3,2$.

\section{Retour des chaleurs après la mise-bas et influence raciale.}

TABLEAU VIII

\begin{tabular}{|c|c|c|}
\hline \multirow{2}{*}{$\begin{array}{c}\text { Temps b́coulé } \\
\text { (jours) }\end{array}$} & \multicolumn{2}{|c|}{ Nombre d'observations } \\
\hline & Juments locales & Axabe-Barbe \\
\hline 41 & 1 & $\vdots$ \\
\hline 35 & 1 & 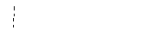 \\
\hline 21 & 1 & \\
\hline 16 & 2. & \\
\hline 14 & 2 & \\
\hline 13 & 2 & 1 \\
\hline 12 & & 1 \\
\hline 11 & 1 & : \\
\hline 10 & 3 & 1 \\
\hline 9 & 4 & 1 \\
\hline 8 & 4 & 1 \\
\hline 7 & 3 & 3 \\
\hline 6 & 3 & 1 \\
\hline 5 & 4 & \\
\hline Total & 31 & 9 \\
\hline Koyerne & 11,3 & 8,8 \\
\hline $\begin{array}{l}\text { Ereur de la } \\
\text { moyenne }\end{array}$ & 2,9 & 1,6 \\
\hline
\end{tabular}

Une fois de plus, l'interprétation statistique montre que le comportement des deux races est sensiblement le même. 


\section{IV. - CONCLUSION}

Les premières manifestations sexuelles apparaissent aux environs $\mathrm{du} 7^{\mathrm{e}}$ mois, tant pour les pouliches du pays que pour les pouliches de race améliorée.

- La durée des chaleurs est en moyenne de 6 à 7 jours, avec un intercestrus de 15 jours.

- La gestation s'échelonne sur 11 mois environ.

- La réapparition des chaleurs après la mise-bas s'effectue en moyenne vers le $12^{\mathrm{e}}$ jour.
- L'âge, de même que le facteur racial n'apportent aucune modification physiologique du tractus génital.

- Enfin, si l'on procède par comparaison avec les juments élevées en Europe, il apparait que les. juments entretenues en milieu tropical obéissent sensiblement aux mêmes lois. Seule, la puberté semble, chez ces dernières, plus précoce de quelques mois et la période d'ancestrus saisonnier généralement observée en milieu tempéré est ici inexistante, l'activité générale se manifestant sans interruption d'un bout de l'année à l'autre.

\section{SUMMARY}

\section{Note on Sexual Behaviour of Mares in Tropical Conditions.}

The sexual behaviour of 24 mares (18 local breeds and 6 arab $\mathrm{x}$ barb crosses) was observed at the Dahra Experimental Station, Senegal, French West Africa, from 1952 to 1955. It was shown that the first sexual manifestations take place as from the 7th month of age while the duration of heat varies from 6 to 7 days with an inter-oestrus period lasting for 15 days. Gestation lasts about 11 months and animals return to heat 12 days after foaling. Neither breed nor age have any influence on the physiological aspect of the genital tract. In tropical countries, puberty seems to appear a few months earlier than under temperate climates and in the former countries there is no seasonal anoestrus. 